\title{
BioéthiqueOnline
}

\section{Facteurs influençant la multiplication de traitements en physiothérapie : une analyse thématique de la jurisprudence québécoise}

\section{Maude Laliberté}

Volume 6, 2017

URI : https://id.erudit.org/iderudit/1044610ar

DOI : https://doi.org/10.7202/1044610ar

Aller au sommaire du numéro

\section{Éditeur(s)}

BioéthiqueOnline

ISSN

1923-2799 (numérique)

Découvrir la revue

Citer cet article

Laliberté, M. (2017). Facteurs influençant la multiplication de traitements en physiothérapie : une analyse thématique de la jurisprudence québécoise. BioéthiqueOnline, 6. https://doi.org/10.7202/1044610ar
Résumé de l'article

La physiothérapie est touchée par un problème de multiplication de traitements; la fréquence des traitements ou leur durée peuvent être inappropriées ou disproportionnées face aux besoins du patient. La multiplication de traitements peut avoir des conséquences financières, physiques, psychologiques et sociales. Pour explorer les facteurs influençant la fréquence et la durée des traitements dans les situations de multiplication de traitements, une analyse thématique de la jurisprudence a été réalisée. Certains facteurs cliniques et non cliniques influençant la fréquence et la durée des traitements ont pu être identifiés par cette analyse thématique de la jurisprudence. Les facteurs cliniques impliquent que l'allocation des ressources soit guidée par la condition et l'évolution du patient. Les facteurs non cliniques peuvent inclure la pression des employeurs, les demandes des autres professionnels de la santé ou les conflits d'intérêts de type financier. Cette analyse thématique de la jurisprudence est une première étape pour comprendre ce qui motive les décisions cliniques d'allocation des ressources des professionnels de la physiothérapie. Cette démarche est essentielle pour mettre en place des politiques et des normes de pratique soucieuses du contexte de pratique et des normes éthiques, déontologiques et légales qui animent la profession.
Droits d'auteur @ Maude Laliberté, 2017

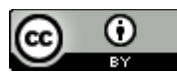

Ce document est protégé par la loi sur le droit d'auteur. L’utilisation des services d'Érudit (y compris la reproduction) est assujettie à sa politique d'utilisation que vous pouvez consulter en ligne. 


\section{Facteurs influençant la multiplication de traitements en physiothérapie: une analyse thématique de la jurisprudence québécoise}

ARTICLE (RÉVISION PAR LES PAIRS / PEER-REVIEWED) Maude Laliberté $1,2,3,4$

Reçu/Received: 29 Jul $2016 \quad$ Publié/Published: 18 Apr 2017
Éditrices/Editors: Vanessa Chenel \& Aliya Affdal
Évaluateurs externes/Peer-Reviewers: Marie-Josée Drolet \& Mélanie Bourassa

2017 M Laliberté, Creative Commons Attribution 4.0 International License

\section{Résumé}

La physiothérapie est touchée par un problème de multiplication de traitements; la fréquence des traitements ou leur durée peuvent être inappropriées ou disproportionnées face aux besoins du patient. La multiplication de traitements peut avoir des conséquences financières, physiques, psychologiques et sociales. Pour explorer les facteurs influençant la fréquence et la durée des traitements dans les situations de multiplication de traitements, une analyse thématique de la jurisprudence a été réalisée. Certains facteurs cliniques et non cliniques influençant la fréquence et la durée des traitements ont pu être identifiés par cette analyse thématique de la jurisprudence. Les facteurs cliniques impliquent que l'allocation des ressources soit guidée par la condition et l'évolution du patient. Les facteurs non cliniques peuvent inclure la pression des employeurs, les demandes des autres professionnels de la santé ou les conflits d'intérêts de type financier. Cette analyse thématique de la jurisprudence est une première étape pour comprendre ce qui motive les décisions cliniques d'allocation des ressources des professionnels de la physiothérapie. Cette démarche est essentielle pour mettre en place des politiques et des normes de pratique soucieuses du contexte de pratique et des normes éthiques, déontologiques et légales qui animent la profession.

\section{Mots clés}

physiothérapie, multiplication de traitements, jurisprudence, analyse thématique, allocation des ressources

\section{Abstract}

Physical therapy is affected by a problem of multiplication of treatments; the frequency and the length of treatments can be inappropriate or disproportionate in the face of a patient's needs. The multiplication of treatments can have financial, physical, psychological and social consequences. To explore the factors that influence the frequency and the total treatment duration in situations where there is a multiplication of treatments, a thematic analysis of the jurisprudence was conducted. Clinical and non-clinical factors influencing the frequency and the treatment duration were identified. Clinical factors mean that resource allocation is guided by the condition and the evolution of the patient. Non-clinical factors can include: pressure from the employer, requests of other health professionals or financial conflict of interests. This thematic analysis of the jurisprudence is a first step to understanding what motivates the clinical decisions of physical therapists regarding the allocation of physical therapy resources. This approach is essential to establishing policies and standards of practice that are attentive to the context of practice and the ethical, deontological and legal standards that shape the profession.

\section{Keywords}

physical therapy, multiplication of treatments, jurisprudence, thematic analysis, resource allocation

\section{Responsabilités des évaluateurs externes}

Les évaluations des examinateurs externes sont prises en considération de façon sérieuse par les éditeurs et les auteurs dans la préparation des manuscrits pour publication. Toutefois, être nommé comme examinateur n'indique pas nécessairement l'approbation de ce manuscrit. Les éditeurs de BioéthiqueOnline assument la responsabilité entière de l'acceptation finale et la publication d'un article.

\section{Peer-reviewer responsibilities}

Reviewer evaluations are given serious consideration by the editors and authors in the preparation of manuscripts for publication. Nonetheless, being named as a reviewer does not necessarily denote approval of a manuscript; the editors of BioéthiqueOnline take full responsibility for final acceptance and publication of an article. 
Affiliations des auteurs / Author Affiliations

${ }^{1}$ École de réadaptation, Faculté de médecine, Université de Montréal, Montréal, Canada

${ }^{2}$ Programmes de bioéthique, Département de médecine sociale et préventive, École de santé publique de l'Université de Montréal

${ }^{3}$ Membre étudiant de l'Institut de recherche en santé publique de l'Université de Montréal (IRSPUM)

${ }^{4}$ Membre étudiant du Centre de recherche interdisciplinaire en réadaptation du Montréal métropolitain (CRIR)

\section{Correspondance / Correspondence}

Maude Laliberté, maude.laliberte@umontreal.ca

\section{Remerciements}

Je tiens à remercier Tatiana Orozco pour son aide précieuse lors de la collecte et l'analyse de données, ainsi que Bryn Williams-Jones et Debbie E. Feldman pour leurs commentaires constructifs tout au long du processus d'analyse et de rédaction. Je tiens finalement à remercier Josée Lemoignan, Geneviève McCready, et les éditeurs de BioéthiqueOnline pour leurs suggestions visant à bonifier le texte.

\section{Conflit d'intérêts}

Maude Laliberté a antérieurement occupé un poste de syndic-adjointe à l'Ordre professionnel de la physiothérapie du Québec et d'éditrice pour BioéthiqueOnline. Son directeur de thèse, Bryn WilliamsJones (éditeur en chef de la revue), n'a pas participé à l'évaluation du manuscrit.

\section{Acknowledgements}

I would like to thank Tatiana Orozco for her precious help for the data collection and analysis, as well as Bryn WilliamsJones and Debbie E. Feldman for their helpful comments throughout the analysis and the writing process. Finally, I also want to thank Josée Lemoignan, Geneviève McCready, and the BioéthiqueOnline editors for their helpful suggestions.

\section{Conflicts of Interest}

Maude Laliberté was previously assistant syndic at the Ordre professionnel de la physiothérapie du Québec and Editor at BioéthiqueOnline. Her PhD supervisor, Bryn Williams-Jones (Editor-in-chief of the journal), was not involved in the review of the manuscript.

\section{Introduction}

La multiplication de traitements (aussi appelé surtraitement) est un problème qui a été documenté en physiothérapie [1,2], tout comme dans plusieurs champs de pratique de la médecine [3-6]. On parle de multiplication de traitements lorsque le nombre de traitements, soit la fréquence ou la durée des traitements, est inappropriées aux besoins du patient ou ou disproportionnées par rapport à ce qui est requis pour atteindre l'objectif clinique visé [7]. Bien que le nombre moyen de traitement par patient varie entre 5 et 13 dans les cliniques externes de physiothérapie [2,8-11], on observe qu'ils peut s'élever jusqu'à 330 dans certains cas [8]. Selon le bureau du syndic de l'Ordre professionnel de la physiothérapie du Québec (OPPQ) ${ }^{1}$, le Québec n'est pas épargné par ce problème [12]. Effectivement, selon une revue de presse québécoise, les patients dont le coût des soins est défrayé par la Commission des normes, de l'équité, de la santé et de la sécurité du travail (CNESST) ${ }^{2}$, semblent avoir une intensité de traitement plus élevée en terme de fréquence et de durée que ceux qui ne bénéficient pas de cette couverture, et ce, sans égard à leurs besoins cliniques [13]. Ainsi, deux patients qui se présentent en physiothérapie avec le même problème pourraient recevoir un nombre de traitements différent.

Les conséquences de la multiplication de traitements peuvent être sociales et financières $[1,6,14,15]$. En plus des coûts directs pour les traitements, il s'agit d'un gaspillage de ressources qui pourraient être mieux utilisées ailleurs [14]. De plus, il y a potentiellement des conséquences physiques et psychologiques iatrogéniques associées aux modalités de traitements elles-mêmes $[1,6,12,14,16]$. Par exemple, le bureau du syndic de l'OPPQ soutient dans un avis envoyé à tous ses membres en

\footnotetext{
${ }^{1}$ Au Québec, les professionnels de la physiothérapie, soit les physiothérapeutes et les techniciens en réadaptation physique (T.R.P.), doivent être membres de l'OPPQ. L'OPPQ a pour mandat d'assurer la protection du public à travers la surveillance et le contrôle de la pratique professionnelle de ses membres. Ce mandat lui est conféré par le Code des professions (L.R.Q., chapitre c-26).

${ }^{2}$ Organisme anciennement appelé la Commission de la santé et de la sécurité du travail (CSST).
} 
2010, qu'une multiplication de traitements peut entrainer la chronicisation d'une condition physique et que cette pratique pose un risque pour la réputation de la profession [12].

Les décisions d'allocation des ressources en lien avec la fréquence ou de la durée optimale des traitements fluctuent beaucoup et les causes de cette disparité ne sont pas bien documentées. Effectivement, plusieurs facteurs peuvent influencer l'allocation des ressources ${ }^{3}$ et ainsi, le problème de multiplication de traitements. Par exemple, le processus décisionnel menant au congé d'un patient est complexe et n'est pas basé sur des stratégies prédéfinies applicables à tous les cas en physiothérapie [17,18]. Baldwin [1] plaide qu'un retour à un seuil fonctionnel et à une bonne qualité de vie pourrait être un critère intéressant pour la pratique en physiothérapie. Cependant, pour des patients avec des conditions similaires, certains physiothérapeutes vont traiter jusqu'à l'atteinte du fonctionnement de base antérieur, d'autres jusqu'à ce qu'à ce que le degré fonctionnel soit adéquat, et certains jusqu'au rétablissement complet [18].

En physiothérapie, il y a une absence de repères spécifiques relatifs à l'allocation des ressources, qui résulterait d'une réflexion collective et exprimerait un consensus ou une norme de pratique reconnue. Les guides de pratique donnent des balises sur les modalités thérapeutiques, mais demeurent lacunaires sur la fréquence ou la durée de traitements qui seraient optimales. Le code de déontologie des physiothérapeutes et des thérapeutes en réadaptation physique (L.R.Q., C. C -26, a. 87) énonce à cet effet des normes minimales en ce qui concerne l'allocation des ressources. Par exemple, l'article 6 stipule que «le membre doit exercer sa profession selon les normes généralement reconnues par la science et la pratique de la physiothérapie » et l'article 19 stipule que " le membre doit éviter de poser ou de multiplier sans motif raisonnable des actes professionnels dans l'exercice de sa profession et doit s'abstenir de poser un acte inapproprié ou disproportionné au besoin de son client ». De plus, l'article 20 du code de déontologie oblige à consulter un autre professionnel si l'état du patient l'exige : "Le membre doit reconnaître en tout temps le droit du client de consulter un autre membre, un autre professionnel ou une autre personne compétente. Si l'état du client l'exige, le membre doit consulter un autre membre, un autre professionnel ou une autre personne compétente ou le diriger vers l'une de ces personnes. »

Pour documenter les facteurs influençant la fréquence et la durée des traitements dans les situations de multiplication de traitements, une analyse thématique de la jurisprudence a été réalisée. La jurisprudence est composée de l'ensemble des décisions disciplinaires. Effectivement, chaque ordre professionnel québécois a un conseil de discipline qui a le mandat d'entendre les plaintes formulées contre un professionnel soupçonné d'avoir commis des infractions aux règlements de son ordre professionnel. Les décisions disciplinaires rendues incluent certains éléments de la preuve permettant de déterminer la conformité ou la non-conformité de la pratique du professionnel ainsi que les sanctions imposées, le cas échéant $[19,20]$. La preuve peut inclure des informations de diverses sources (avis d'experts, littérature scientifique). Ainsi, cette analyse thématique de la jurisprudence a pour but de définir ce qu'est une multiplication de traitements pour le conseil de discipline de l'OPPQ et de quelle façon elle est objectivée. Elle vise également à identifier certains facteurs contribuant à ce phénomène. Effectivement, bien que chaque décision disciplinaire cible le comportement individuel d'un professionnel dans un contexte particulier, la jurisprudence nous permettra d'observer certains facteurs systémiques au problème de multiplication de traitements. Ce faisant, nous pourrons identifier certaines avenues de solutions pour mieux gérer l'allocation des ressources en physiothérapie.

3 Dans cet article, l'allocation des ressources fait référence aux décisions concernant la meilleure façon pour un physiothérapeute ou un département de physiothérapie d'allouer les ressources pour un patient ou pour un groupe de patients. 


\section{Méthode}

Cette étude a utilisé un devis qualitatif considérant qu'il vise à mieux comprendre un phénomène où plusieurs variables sont encore peu documentées [21,22]. La méthode choisie fut l'analyse thématique descriptive qui permet d'identifier, d'analyser et de rapporter des thèmes qui décrivent un phénomène [23,24]. Cette méthode permet d'organiser les données pour que leur description minutieuse contribue à l'interprétation d'un phénomène [24].

L'analyse de la jurisprudence a été effectuée à la fin février et au début mars 2016, sur le site de I'Institut canadien d'information juridique (CanLii.org) dans la section dédiée au Tribunal administratif du conseil de discipline de l'OPPQ. Le moteur de recherche de CanLii.org répertorie les décisions depuis 2002 pour le Tribunal administratif du conseil de discipline de l'OPPQ.

Une première étape de la collecte de décisions disciplinaires a été faite avec une recherche ciblée par mots-clés (multip* ET acte*). Cette recherche a permis de recueillir 87 décisions disciplinaires. De ces décisions, 2 étaient des doublons et 44 ne concernaient pas la multiplication de traitements. Par la suite, une deuxième étape a été faite avec une recherche par mots-clés avec l'article 19 du code de déontologie ([19] ET [multipli* ou disprop*]) qui a permis d'identifier 51 décisions. De celles-ci, 46 étaient des doublons de notre première étape de collecte, 4 ont été exclues, car elles ne concernaient pas la multiplication de traitements. Une troisième étape a été faite avec une recherche par mots-clés ([multipli* ou disprop*] et [3.02.06]) et celle-ci a permis d'identifier 18 décisions, avec l'article 3.02.06 de l'ancienne version du code de déontologie, mais toutes les décisions disciplinaires identifiées étaient des doublons des recherches précédentes. Ainsi, 42 décisions ont été analysées au total (voir figure 1). La stratégie de recherche a été validée auprès d'une bibliothécaire de la bibliothèque de la faculté de Droit de l'Université de Montréal.

Figure 1 - Diagramme illustrant les phases de la collecte de données

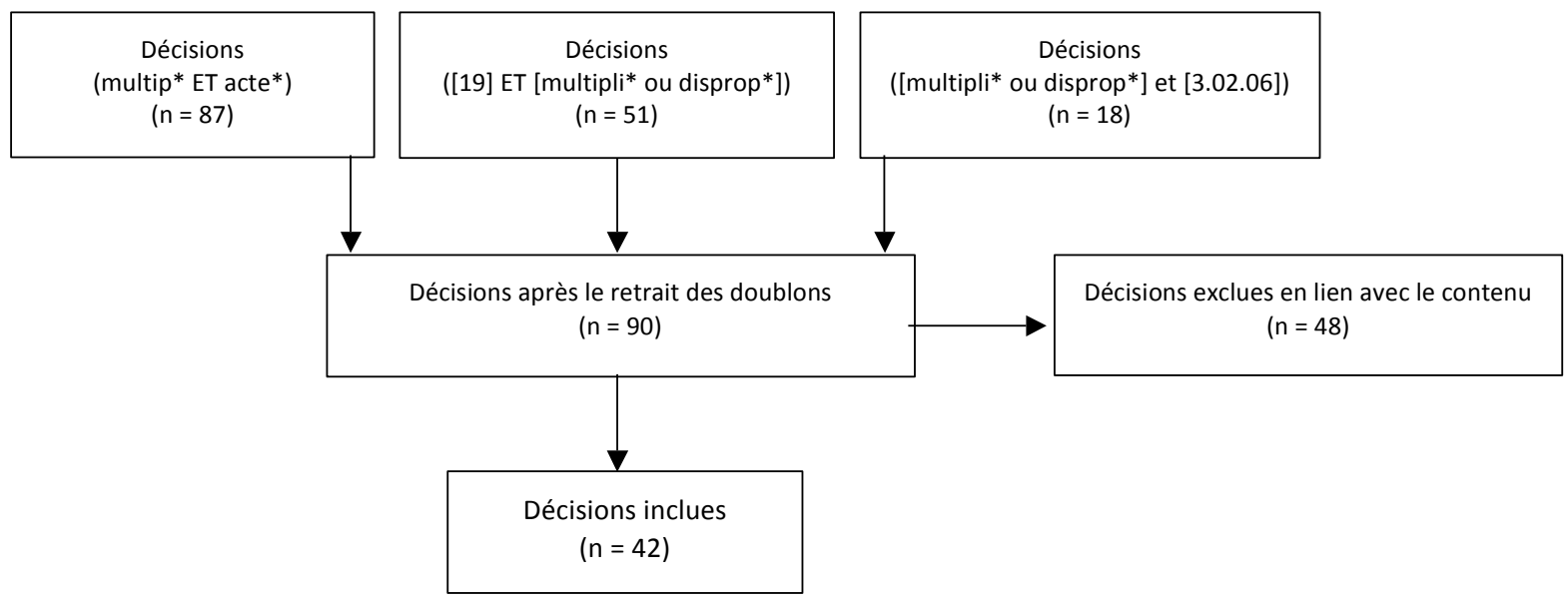

Les décisions disciplinaires retenues ont été analysées à l'aide d'une grille qui inclut des informations plus descriptives comme le code d'identification, la date des incidents, l'âge et la profession du professionnel de la physiothérapie visé (physiothérapeute ou techniciens en réadaptation physique, T.R.P.). Des informations liées à la faute et la culpabilité, les facteurs aggravants et atténuants identifiés par les différentes parties, la définition donnée au phénomène de la multiplication de traitements ainsi que la méthode visant son objectivisation ont aussi été inclues dans notre analyse. L'extraction a d'abord été faite par une assistante de recherche puis l'ensemble des décisions a été révisé et bonifié par l'auteure. L'analyse thématique a été utilisée pour identifier les relations et les 
thèmes récurrents parmi les données analysées pour répondre à l'objectif de recherche. Les résultats de l'analyse ont été validés par deux chercheurs de l'Université de Montréal. ${ }^{4}$

\section{Résultats}

D'abord, une définition sommaire du phénomène de la multiplication de traitements, sous l'angle du conseil de discipline de l'OPPQ (ci-après le Conseil), sera présentée. De plus, les moyens utilisés pour établir la preuve dans les décisions disciplinaires liées à la multiplication de traitements seront décrits. Ensuite, les divers facteurs contribuant à la multiplication de traitements identifiés par l'analyse thématique de la jurisprudence seront exposés.

\section{Définition de la multiplication de traitements et moyens utilisés pour établir la preuve}

Selon le Conseil, la multiplication de traitements consiste en une plus grande répétition de traitements que ce qui est requis par la condition ou l'évolution clinique du patient. Ainsi, selon le Conseil, ces traitements sont qualifiés d'inappropriés, inutiles et/ou excessifs. Effectivement, dans les décisions analysées, un très grand nombre de traitements a pu être prodigué à certains patients (plusieurs centaines de traitements pour un même patient), dénotant le caractère excessif de cette pratique. Par exemple, certains professionnels de la physiothérapie ont prodigué plus de 250 traitements, parfois même jusqu'à 494 traitements, sur des périodes de temps variant de 13 à 24 mois [25-28].

Dans les décisions analysées, le Conseil a utilisé divers moyens pour déterminer s'il y avait multiplication de traitements ou non. Par exemple, pour pouvoir déterminer les normes de pratiques actuelles, le Conseil a parfois fait appel à l'avis d'un expert [25,29]. Les experts, dans les décisions analysées, étaient des cliniciens reconnus détenant une spécialisation en lien avec les thèmes abordés. Les écrits scientifiques ont aussi été invoqués, à la fois pour préciser les normes de pratiques [29,30], mais aussi pour soulever le manque ou l'imprécision des données probantes pour baser la pratique clinique $[29,30]$. Par exemple, les écrits suggèrent que l'ultrason soit utilisé sans interruption un maximum de 15 traitements selon certains auteurs, et pouvant aller jusqu'à 30 selon d'autres [29]. Finalement, l'outil le plus régulièrement utilisé est le dossier clinique, où sont consignées les notes du clinicien [25,27,29,31-33]. Celles-ci permettent d'expliciter le raisonnement à la base des décisions cliniques d'allocation des ressources des professionnels de la physiothérapie.

\section{Facteurs contribuant à la multiplication de traitements}

De façon générale, on constate que les décisions disciplinaires impliquaient plus régulièrement des femmes ( 27 femmes et 15 hommes), des T.R.P. (23 T.R.P. et 19 physiothérapeutes) et des cliniciens (36 cliniciens et 6 propriétaires ou gestionnaires de cliniques privées). Le niveau d'expérience des professionnels variait beaucoup et n'était pas consigné de façon constante ( 5 avaient moins de 3 ans d'expérience; 10 avaient entre 3 et 8 ans d'expérience; 4 avaient plus de 21 ans d'expérience; dans 23 cas, cette information n'était pas disponible). Les décisions concernaient non seulement des cliniciens (physiothérapeutes et T.R.P.) pour leur pratique individuelle de multiplication de traitements, mais également des collègues qui n'ont pas dénoncé ces pratiques [30,34] et des employeurs des thérapeutes qui ont encouragé les pratiques ou qui n'ont pas agi pour les empêcher [35,36], et ce, peu importe s'ils retiraient ou non un avantage personnel (financier ou autre) de ces pratiques.

\footnotetext{
4 Bryn Williams-Jones est professeur et directeur des programmes de bioéthique; il est spécialisé dans les questions de conflits d'intérêts. Debbie E. Feldman est professeur à l'École de réadaptation et se spécialise dans les questions d'accès aux services de physiothérapie.
} 
ONLINE

Tableau 1 - Facteurs contribuant à la multiplication de traitements

\begin{tabular}{|c|c|}
\hline Niveau & Facteurs \\
\hline Professionnel (micro) & $\begin{array}{l}\text { - Type de modalités de traitement (modalités passives) } \\
\text { - Traitement non-indiqué en fonction de la condition du patient } \\
\text { - Stabilité de la condition du patient malgré les traitements }\end{array}$ \\
\hline Département (méso) & $\begin{array}{l}\text { - Cliniques privées } \\
\text { - Comportement de l'employeur (encourage la pratique, } \\
\text { pression directe, n'empêche pas la pratique) } \\
\text { - Comportement des collègues (ne dénoncent pas la situation, } \\
\text { continuent les traitements inutiles) } \\
\text { - Comportement des médecins traitants qui prescrivent la } \\
\text { poursuite des traitements }\end{array}$ \\
\hline Institutionnel (macro) & - Normes ou politiques des agents payeurs (ex. : CNESST) \\
\hline
\end{tabular}

Certains facteurs influençant la multiplication de traitements sont liés directement aux décisions du professionnel face au traitement clinique de son patient. D'abord, on constate que les multiplications de traitements impliquent généralement des modèles récurrents d'application de modalités passives, administrées en série, comme la chaleur, le massage, l'ultrason, le neurostimulateur électrique transcutané (TENS), le microcourant, le courant interférentiel, le bain-tourbillon et les tractions. En plus de ne pas améliorer la condition du patient, ces traitements multipliés pourraient lui nuire [25,2931]. Par exemple, il y a des effets potentiellement néfastes à recevoir des traitements inefficaces sur une longue période, dont un risque de chroniciser le problème. Aussi, des ultrasons administrés sans interruption avec un dosage excessif pourraient être nocifs pour un patient $[25,29,31]$.

Il est essentiel que les traitements soient indiqués pour la condition du patient. Par exemple, il a été reconnu comme inapproprié d'utiliser une modalité thérapeutique comme un courant électrique de type iontophorèse ${ }^{5}$ pour réduire les calcifications, soit dissoudre les dépôts de calcium, chez un patient ayant un rapport de radiologie normal sans dépôt de calcium [37].

II doit y avoir un effet positif sur la condition du patient avec le traitement [32]. Ainsi, lorsque le patient est stable et qu'il n'y a plus d'évolution, c'est-à-dire qu'il ne montre plus de gains réels ou d'amélioration, on considère que le plan de traitement devrait être modifié ou que les traitements devraient cesser [25]. D'ailleurs, une décision indique que « les traitements faits [...] auraient dû être suspendus au moment où c'était clair que le patient n'évoluait pas » [31]. La stabilisation de la condition du patient est évaluée à partir de différents éléments comme l'évaluation objective, l'état initial du patient et son évolution, la progression des traitements [25]. Alors, le comportement attendu du clinicien serait de réévaluer régulièrement l'état de son patient, et ce, en fonction des données objectives telles que les tests cliniques et son jugement clinique, puis documenté au dossier [29]. Cette réévaluation permet au professionnel de valider l'efficacité des modalités thérapeutiques et de modifier le plan de traitement, s'il y a lieu. En outre, il peut être indiqué de suspendre les traitements de physiothérapie, de référer le patient à un collègue ayant une expertise plus spécifique ou de suggérer au médecin référent l'arrêt des traitements [26,29,38-47]. II faut toutefois préciser que dans certaines situations, la poursuite des traitements en physiothérapie est justifiée même en l'absence d'une évolution positive du patient. Ainsi, un patient pourrait nécessiter une plus longue période de traitement, et ce jusqu'à la consolidation de sa blessure [27].

Deuxièmement, certains facteurs influençant la multiplication de traitements sont liés aux pratiques départementales. II importe de souligner que la totalité des cas de multiplication de traitements se sont déroulés dans des milieux privés $(n=42 / 42)$. Certains cliniciens qui remplaçaient des collègues

\footnotetext{
${ }^{5}$ L'iontophorèse est une modalité d'électrothérapie qui consiste en un courant électrique continu et constant qui est utilisé pour traiter des problèmes d'inflammation, de douleur ou de calcification (source : Électrologic).
} 
pendant des vacances ont été reconnus coupables de multiplication de traitements même s'ils n'étaient pas responsables de l'ensemble des traitements administrés [38]. Ainsi, le Conseil montre qu'il est essentiel de considérer les traitements préalables et d'utiliser le jugement clinique lors de chacun des traitements. Certains propriétaires de clinique ont mis une pression directe ou indirecte sur leurs employés pour prolonger la durée totale et la fréquence des traitements $[28,32,40,41,48]$. Cependant, le Conseil a statué que le fait qu'un employeur ait demandé de multiplier les traitements ne constituait pas une excuse valable pour justifier le comportement de l'employé [28,32,40,41,48]. Les médecins traitants peuvent également encourager cette pratique en exigeant la poursuite de traitements en physiothérapie malgré que le professionnel désire les cesser [25,49]. Le Conseil, à travers maintes décisions, lance un message clair: les physiothérapeutes et les T.R.P. sont des professionnels autonomes dans leur champ d'exercice et ils sont responsables de mettre fin au traitement lorsque les objectifs sont atteints ou lorsqu'il y a une stabilisation de la condition du patient. Ils ne peuvent pas, sous aucun prétexte, accepter de continuer des traitements inutilement même si cela est exigé par un tiers qu'il s'agisse d'un employeur $[28,32,40,41,48]$ ou de tout autre professionnel de la santé tel que le médecin traitant $[25,49]$.

Troisièmement, certains facteurs institutionnels comme les normes ou les politiques des agents payeurs semblent influencer la multiplication de traitements. Près de $40 \%$ des décisions analysées impliquaient des agents payeurs comme la CNESST $(n=16 / 42)$. Le Conseil rappelle d'ailleurs que les motivations économiques pouvant sous-tendre de façon implicite ou explicite la décision clinique du professionnel de multiplier les traitements (ex.: par désir de rentabiliser une clinique) sont inadmissibles car elles donnent un aspect de commercialité à la profession [35]. La multiplication de traitements entache la confiance du public et ternit la crédibilité et l'image de la profession [39].

\section{Discussion}

Cette analyse de la jurisprudence a permis de mettre en lumière certains facteurs qui influencent la fréquence et la durée des traitements dans les situations de multiplication de traitements.

D'abord, des facteurs peuvent être liés aux décisions cliniques du professionnel. Ceux-ci impliquent que l'allocation des ressources, soit la fréquence et la durée des traitements, soit déterminée par la condition ou l'évolution du patient. Ainsi, la condition du patient doit s'améliorer avec les traitements de physiothérapie et ceci doit être documenté au dossier. Or, on peut constater qu'il n'y a pas de " recettes » et qu'il pourrait survenir certains cas où un grand nombre de traitements est utile, voire même souhaitable (ex. : jusqu'à la consolidation d'une blessure [27]). Dans les circonstances où la décision du thérapeute s'écarte des normes professionnelles établies, la justification clinique motivant cette décision doit être établie et rigoureusement consignée dans le dossier du patient.

Cette analyse de la jurisprudence a également permis de mettre en lumière certains facteurs en lien avec le département de physiothérapie ou encore des facteurs institutionnels qui peuvent influencer la fréquence et la durée des traitements dans les situations de multiplication de traitements. Ceci est cohérent avec les écrits alors que les actions non conformes aux standards professionnelles peuvent généralement être expliquées à la fois par des raisons individuelles (ex. : comportement et tempérament du professionnel) et par des dynamiques sociales (ex. : structure organisationnelle) [70]. Ainsi, dans la jurisprudence, on peut constater que la pression des employeurs ou les demandes de maintien des traitements par un autre professionnel de la santé (comme le médecin traitant) peuvent avoir une influence sur l'allocation des ressources. De plus, les milieux de soins privés à but lucratif sont directement en cause. Cette rationalité économique peut entrer en conflit avec les valeurs des professionnels; les systèmes de paiement peuvent influencer le comportement des professionnels [50-52] ou des institutions [53,54]. Aussi, la jurisprudence impliquait des agents payeurs comme la CNESST dans près de $40 \%$ des cas. Au Québec, les lésions de type musculosquelettique touchent plus de 30000 travailleurs annuellement et un grand nombre de patients couverts par la CNESST sont suivis en physiothérapie [55]. Le rôle complexe des agents 
payeurs dans les entreprises privées soulève la question des conflits d'intérêts en physiothérapie. Effectivement, les systèmes de paiements peuvent créer des incitatifs et des conflits d'intérêts qui influencent le raisonnement clinique, les décisions organisationnelles et le comportement des professionnels et de leurs gestionnaires en lien avec l'allocation des ressources.

Un conflit d'intérêts dans un contexte professionnel est une situation dans laquelle une personne a un intérêt privé ou personnel, financier ou non financier, qui influence indûment l'exercice objectif de ses fonctions officielles [56,57]. Un conflit d'intérêt peut être apparent, réel ou potentiel. Un conflit d'intérêts représente un ensemble de circonstances qui entraîne un risque qu'un intérêt secondaire interfère avec le jugement ou les actions relatives à un intérêt qui devrait être primaire [58,59]. Un point central de la notion de conflit d'intérêts relative aux professionnels de la santé se retrouve dans la relation fiduciaire, c'est-à-dire le lien de confiance entre le professionnel et son patient, basé sur l'attente que les intérêts du patient soient priorisés par rapport à ceux du professionnel [60]. Dans cette relation thérapeutique inégalitaire, le patient est vulnérable car le professionnel détient une expertise spécifique dont il est dépendant. II est donc essentiel que le professionnel agisse avec prudence, bonne foi, loyauté et honnêteté dans le meilleur intérêt de son patient et pour honorer ce lien de confiance [61]. Ainsi, cette relation de confiance s'avère menacée par un intérêt qui interfère avec l'exercice du jugement du professionnel.

En physiothérapie, il peut y avoir un conflit entre le meilleur intérêt du patient d'accéder et de bénéficier de services professionnels appropriés à sa condition (et la responsabilité du professionnel d'agir dans le meilleur intérêt du patient) et l'intérêt financier de la clinique ou du professionnel. Cette situation est démontrée dans les écrits qui indiquent que les patients ayant subi une arthroplastie du genou aux États-Unis ont eu deux fois plus de traitements en moyenne lorsqu'ils étaient traités dans une clinique de physiothérapie où leur chirurgien orthopédiste avait des actions que lorsqu'ils étaient pris en charge dans une clinique indépendante [62]. Si ces conflits d'intérêts ne sont pas bien gérés, ils peuvent entraîner des torts significatifs allant au-delà de la rupture du lien de confiance avec le patient en nuisant directement au traitement du patient. Ceux-ci peuvent miner la réputation du physiothérapeute, de l'institution qu'il représente et même celle de la profession en général. De plus, les conflits d'intérêts peuvent mettre en péril les relations professionnelles et donc le bon fonctionnement des équipes de travail [57,59]. À cet égard, le Conseil rappelle que les motivations économiques sont inadmissibles, ternissant l'image de la profession en lui donnant un aspect de lustre et de commercialité. II devient donc essentiel d'identifier les mesures appropriées pour encadrer les conflits d'intérêts et bien gérer ceux qui ne peuvent pas être évités.

Plusieurs stratégies doivent être mises en place de façon parallèle pour gérer un problème aussi complexe que la multiplication de traitements en physiothérapie. Le but ultime est que chaque acteur modifie sa pratique de façon cohérente avec les valeurs de la profession face à l'allocation des ressources. D'abord, il est essentiel d'éduquer et de sensibiliser les professionnels de la physiothérapie [3]. À ce jour, I'OPPQ a mis en place des leviers d'actions distincts, mais intrinsèquement reliées, pour sensibiliser les professionnels de la physiothérapie. Par exemple, le Conseil est à la fois une autorité qui permet de sanctionner le comportement d'un membre ayant dérogé à ses obligations professionnelles, et un incitatif permettant de donner l'exemple pour persuader les membres de se conformer aux normes de pratique [63]. Le bureau du syndic de l'OPPQ a également émis un avis en lien avec la multiplication de traitements à ses membres en 2010 pour rappeler certains principes de vigilance et de responsabilité professionnelle [12]. Cette initiative est très importante, car il est démontré que les politiques sur les conflits d'intérêts sont plus efficaces et mieux acceptées quand leurs motivations éthiques sont bien comprises, que lorsqu'elles sont perçues comme imposées par un tiers externe [64].

Parallèlement, la formation continue est un levier d'action pour aider les cliniciens à maintenir leur compétence optimale dans l'exercice de leur profession. D'ailleurs, l'OPPQ favorise l'amélioration des compétences des physiothérapeutes gradués par une politique à cet égard [65]. Une pratique basée 
sur les données probantes nécessite que les physiothérapeutes s'informent des nouvelles pratiques exemplaires. Cependant, il n'existe aucune formation spécifique relative à la gestion des conflits d'intérêts. Favoriser ses propres intérêts n'est pas foncièrement mauvais et est un instinct généralement inconscient, automatique et viscéral, alors que la reconnaissance de nos obligations envers les autres implique un processus beaucoup plus réflexif [66]. Le problème survient si l'intérêt de sa clinique ou ses propres intérêts sont favorisés au détriment de ceux du patient. En physiothérapie, le College of Physiotherapists of Ontario innove en offrant des modules d'autoapprentissage en ligne dédiés à l'éthique [67]. L'enseignement, la formation, et la diffusion d'informations peuvent aider les physiothérapeutes à reconnaître les conflits d'intérêts (financiers et non financiers) et à comprendre leur influence sur leur pratique [68]. Plus il y aura d'individus outillés pour avoir une pratique réflexive face aux conflits d'intérêts, plus la profession aura une culture éthique [69].

Certaines études de Papadakis et collègues ont fait une corrélation entre des sanctions disciplinaires en médecine et des antécédents d'incidents survenus pendant les études universitaires dénotant du non-professionnalisme [71-73]. Notre analyse ne permet pas d'établir le « portrait » d'un professionnel de la physiothérapie qui serait plus enclin à faire de la multiplication de traitements, mais il serait intéressant de se pencher sur cette question.

La multiplication de traitements est un geste non professionnel qui entache la confiance du public. Cependant, la question de l'allocation des ressources est complexe. Les guides de pratiques demeurent lacunaires au regard de la fréquence et de la durée optimale des traitements. Ces mêmes guides de pratique donnent des balises uniquement pour certaines modalités thérapeutiques. L'analyse de la jurisprudence illustre ces limites puisque les écrits scientifiques sont imprécis pour baser la pratique clinique [29,30]. II est essentiel de documenter à l'aide de données probantes l'efficacité des modalités thérapeutiques cliniques, mais également leur application optimale pour obtenir des effets bénéfiques [1]. II serait important de déterminer le seuil à partir duquel il y a des risques dans l'application de ces modalités thérapeutiques auprès d'un patient. Effectivement, la multiplication de traitements implique une surestimation des bénéfices cliniques et une sousestimation des risques à la fois par le professionnel et par le patient [14]. Cependant, comme Carter et collègues (2015) le soulignent, il est essentiel de bien considérer la nature de ces risques et pondérer leur importance réelle à la fois pour le patient, pour le clinicien et pour la société [15]. Par exemple, certaines contre-indications dans l'application du TENS (ex. : utilisation lors d'un diagnostic cancer) demeurent des risques théoriques qui n'ont pas été démontrés par les données probantes [74]. Des critères clairs, justes et transparents, soutenus par des données probantes et par d'autres principes pertinents, peuvent fournir des orientations pour la prise de décisions autour de l'allocation des ressources [75-80], mais ces critères doivent faire l'objet d'un consensus clair dans la profession.

De plus, des incitatifs directs et indirects (attentes, politiques, conventions, pression des agents payeurs, des employeurs ou des collègues) peuvent avoir eu une influence pernicieuse, en créant une nouvelle norme de pratique collective quant à l'allocation des ressources [81,82], entre autres en physiothérapie en lien avec la CNESST [83]. L'impact des diverses politiques devrait être évaluée pour déterminer leur réelle influence sur la pratique [4]. Par exemple, le phénomène de multiplication de traitements pourrait prendre racine dans un contexte de sous-financement avec un écart important entre le montant facturé par la clinique de physiothérapie pour un patient suivi par la CNESST qui est moindre que celui que que les autres patients doivent débourser, suivant la norme du marché [13]. Ainsi, prolonger la durée du suivi et multiplier le nombre de traitements permet d'augmenter la rentabilité des cliniques. Donc, la question suivante se pose : comment bien répartir les ressources en physiothérapie avec des soins de qualité pour aider les patients, tout en étant soucieux des attentes de la société et des contraintes financières? 


\section{Limites de l'étude}

Cette analyse thématique de la jurisprudence ne permet pas d'avoir des données exhaustives sur l'ensemble des facteurs influençant la fréquence et la durée des traitements dans les situations de multiplication de traitements, car l'analyse porte uniquement sur des décisions disciplinaires, soit les cas où un professionnel était soupçonné d'avoir commis des infractions aux règlements de son ordre professionnel. De plus, la décision disciplinaire contient seulement certains éléments de la preuve appuyant l'argumentaire du Conseil pour déterminer la conformité ou la non-conformité de la pratique du professionnel. Ainsi, bien que ces éléments ne puissent pas documenter la pratique idéale, elle permet d'établir les normes minimales associées à la pratique.

\section{Conclusion}

Par cette analyse de la jurisprudence, certains facteurs influençant la fréquence et la durée des traitements ont pu être identifiés. Les facteurs liés aux décisions cliniques du professionnel impliquent que l'allocation des ressources soit guidée par l'évolution clinique favorable du patient en lien avec le traitement. Ces facteurs sont évalués par le jugement clinique du professionnel qui se base sur des tests objectifs. D'autres facteurs en lien avec l'organisation du travail incluant la pression des employeurs, les demandes des autres professionnels de la santé, ou l'implication d'agents payeurs ont également un rôle à jouer. Ceci permet de mettre en cause des conflits d'intérêts potentiels dans les décisions d'allocation des ressources. Les conflits d'intérêts peuvent influencer le jugement clinique et porter atteinte à la confiance du public. II devient donc indispensable de pouvoir les identifier et les gérer adéquatement afin que les patients puissent être traités de façon équitable et en fonction de leurs besoins. Cette analyse de la jurisprudence est une première étape pour comprendre ce qui motive les décisions cliniques des professionnels de la physiothérapie dans l'allocation des ressources en physiothérapie. Cette démarche est essentielle pour mettre en place des politiques et des normes de pratiques soucieuses du contexte de pratique et des normes éthiques, déontologiques et légales qui animent la profession.

\section{Références}

1. Baldwin, J., McKay M.J., Hiller, C.E. on behalf of the 1000 Norms Project Consortium, et al., Defining health and disease: setting the boundaries for physiotherapy. Are we undertreating or overtreating? How can we tell? British Journal of Sports Medicine, 2015. 49(9):1225-1226

2. Swinkels, I.C., et al., What factors explain the number of physical therapy treatment sessions in patients referred with low back pain; a multilevel analysis. BMC Health Services Research, 2005. 5(74).

3. Bhatt, J.R. and L. Klotz, Overtreatment in cancer-is it a problem? Expert Opinion on Pharmacotherapy, 2016. 17(1):1-5.

4. Esserman, L.J., et al., Addressing overdiagnosis and overtreatment in cancer: a prescription for change. The Lancet Oncology, 2014. 15(6):e234-e242.

5. Loeb, S., et al., Overdiagnosis and overtreatment of prostate cancer. European Urology, 2014. 65(6): p. 1046-1055.

6. Karavidas, N.S., Bracing for Adolescent Idiopathic Scoliosis (AIS) and Scheuermann Kyphosis: the issue of overtreatment in Greece. Scoliosis and Spinal Disorders, 2016. 11(Suppl 2):30.

7. Taylor, P.J., Commentary: Overstating overtreatment? BMJ, 1994. 308(6941): 1413-1414.

8. Halfon, P., et al., The effect of patient, provider and financing regulations on the intensity of ambulatory physical therapy episodes: a multilevel analysis based on routinely available data. BMC Health Services Research, 2015. 15(52).

9. Gracey, J.H., S.M. McDonough, and G.D. Baxter, Physiotherapy management of low back pain: a survey of current practice in Northern Ireland. Spine, 2002. 27(4):406-411. 
10. Machlin, S.R., et al., Determinants of utilization and expenditures for episodes of ambulatory physical therapy among adults. Physical Therapy, 2011. 91(7):1018-1029.

11. Davis, M.A., Where the United States spends its spine dollars: expenditures on different ambulatory services for the management of back and neck conditions. Spine, 2012. 37(19):1693-1701.

12. Gauthier, L., La multiplication des traitements en physiothérapie: soyons proactifs! Avis OPPQ, 2010: 1-2.

13. Laliberté, M. and A. Hudon, La difficile interaction entre les physiothérapeutes et les agents payeurs québécois: une analyse éthique médiatique. Éthique \& Santé, 2014. 11(2): 91-99.

14. Gunatilake, S., Overdiagnosis-overtreatment. Ceylon Medical Journal, 2014. 59(3):73-74.

15. Carter, S., et al., The challenge of overdiagnosis begins with its definition. BMJ: British Medical Journal (Online), 2015. 350.

16. Franks, P., C.M. Clancy, and P.A. Nutting, Gatekeeping revisited-protecting patients from overtreatment. New England Journal of Medicine, 1992. 327:424-429.

17. Hudon, A., et al., Quality of physiotherapy services for injured workers compensated by workers' compensation in Quebec: a focus group study of physiotherapy professionals. Healthcare policy=Politiques de sante, 2015. 10(3):32-47.

18. Poulis, I., The end of physiotherapy. Australian Journal of Physiotherapy, 2007. 53(2):71-72.

19. Salvas, M.-F. Le conseil de discipline: la justice par les pairs. Ordre professionnel de la physiothérapie du Québec.

20. Fortier, C., La pierre angulaire du système de justice par les pairs, Ordre des ergothérapeutes du Québec, Editor.

21. Morse, J.M., A review committee's guide for evaluating qualitative proposals. Qualitative Health Research, 2003. 13(6): 833-851.

22. Marshall, C. and G. Rossman, Designing Qualitative Research. 1989: Newbury Park: Sage Publication.

23. Fereday, J. and E. Muir-Cochrane, Demonstrating rigor using thematic analysis: A hybrid approach of inductive and deductive coding and theme development. International journal of qualitative methods, 2006. 5(1):80-92.

24. Braun, V. and V. Clarke, Using thematic analysis in psychology. Qualitative research in psychology, 2006. 3(2):77-101.

25. Physiothérapie (Ordre professionnel de la) c Abdel Rahman 31-07-008, in Conseil de discipline de l'Ordre professionnel de la physiothérapie du Québec. 2008: CanLII 88242.

26. Physiothérapie (Ordre professionnel de la) c Mailhot 31-10-002, in Conseil de discipline de l'Ordre professionnel de la physiothérapie du Québec. 2011: CanLII 93057.

27. Physiothérapie (Ordre professionnel de la) c Bilodeau-Desrochers 31-14-014, in Conseil de discipline de l'Ordre professionnel de la physiothérapie du Québec. 2015: CanLII 70498.

28. Physiothérapie (Ordre professionnel de la) c Jeannot 31-11-019, in Conseil de discipline de l'Ordre professionnel de la physiothérapie du Québec. 2012: CanLII 19837.

29. Physiothérapie (Ordre professionnel de la) c Cloutier 31-05-003 in Conseil de discipline de l'Ordre professionnel de la physiothérapie du Québec. 2006: CanLII 80372

30. Physiothérapie (Ordre professionnel de la) c Amouzegar, 31-08-012 in Conseil de discipline de l'Ordre professionnel de la physiothérapie du Québec. 2009: CanLII 90412.

31. Physiothérapie (Ordre professionnel de la) c Dalencourt 31-08-009, in Conseil de discipline de l'Ordre professionnel de la physiothérapie du Québec. 2009: CanLII 90424.

32. Physiothérapie (Ordre professionnel de la) c Carignan 31-14-004, in Conseil de discipline de l'Ordre professionnel de la physiothérapie du Québec. 2014: CanLII 28097.

33. Physiothérapie (Ordre professionnel de la) c Bednarczyk 31-14-006, in Conseil de discipline de l'Ordre professionnel de la physiothérapie du Québec. 2015: CanLII 45349.

34. Physiothérapie (Ordre professionnel de la) c Alwan 31-10-003, in Conseil de discipline de l'Ordre professionnel de la physiothérapie du Québec. 2011: CanLII 93055.

35. Physiothérapie (Ordre professionnel de la) c Alwan 31-10-004, in Conseil de discipline de l'Ordre professionnel de la physiothérapie du Québec. 2011: CanLII 93050. 
36. Physiothérapie (Ordre professionnel de la) c Ippersiel 31-11-033, in Conseil de discipline de l'Ordre professionnel de la physiothérapie du Québec. 2012: CanLII 26884.

37. Physiothérapie (Ordre professionnel de la) c Nguon 31-05-009, in Conseil de discipline de l'Ordre professionnel de la physiothérapie du Québec. 2006: CanLII 80378.

38. Physiothérapie (Ordre professionnel de la) c Larouche 31-08-003, in Conseil de discipline de l'Ordre professionnel de la physiothérapie du Québec. 2008: CanLII 89888.

39. Physiothérapie (Ordre professionnel de la) c Tobon 31-05-002, in Conseil de discipline de l'Ordre professionnel de la physiothérapie du Québec. 2005: CanLII 78231.

40. Physiothérapie (Ordre professionnel de la) c Robillard 31-11-026, in Conseil de discipline de l'Ordre professionnel de la physiothérapie du Québec. 2012: CanLII 26859.

41. Physiothérapie (Ordre professionnel de la) c Crépeau 31-11-027, in Conseil de discipline de l'Ordre professionnel de la physiothérapie du Québec. 2012: CanLII 26860.

42. Physiothérapie (Ordre professionnel de la) c Kuete 31-10-001, in Conseil de discipline de l'Ordre professionnel de la physiothérapie du Québec. 2011: CanLII 93044.

43. Physiothérapie (Ordre professionnel de la) c Ménard 31-11-030, in Conseil de discipline de l'Ordre professionnel de la physiothérapie du Québec. 2012: CanLII 26861.

44. Physiothérapie (Ordre professionnel de la) c Samson 31-08-010, in Conseil de discipline de l'Ordre professionnel de la physiothérapie du Québec. 2009: CanLII 90421.

45. Physiothérapie (Ordre professionnel de la) c Brien 31-11-029, in Conseil de discipline de l'Ordre professionnel de la physiothérapie du Québec. 2012: CanLII 26846.

46. Physiothérapie (Ordre professionnel de la) c Bilodeau 31-08-004, in Conseil de discipline de l'Ordre professionnel de la physiothérapie du Québec. 2008: CanLII 88249.

47. Physiothérapie (Ordre professionnel de la) c Boyer 31-11-034, in Conseil de discipline de l'Ordre professionnel de la physiothérapie du Québec. 2012: CanLII 26845.

48. Physiothérapie (Ordre professionnel de la) c Labelle 31-14-005, in Conseil de discipline de l'Ordre professionnel de la physiothérapie du Québec. 2014: CanLII 34477.

49. Physiothérapie (Ordre professionnel de la) c Warten 31-13-002, in Conseil de discipline de l'Ordre professionnel de la physiothérapie du Québec. 2013: CanLII 77784.

50. Brody, H., Professionnal medical organizations and commercial conflicts of interest: ethical issues. Annals of Family Medicine, 2010. 8(4): 354-8.

51. Latham, S.R., Ethics in the marketing of medical services. The Mount Sinaï Journal of Medicine, 2004. 71(4):243-50.

52. Chaix-Couturier, C., et al., Effects of financial incentives on medical practice: results from a systematic review of the literature and methodological issues. International Journal for Quality in Health Care, 2000. 12(2):133-142.

53. Rothman, D.J., Academic medical centers and financial conflicts of interest. JAMA, 2008. 299(6):695-7.

54. McGreevey, S., More attention needed to conflicts presented by institutional relationships. WebWire, Feb 132008.

55. CSST, Bâtir l'assurance d'une prévention durable. Plan Stratégique 2010-2014, 2010. Commission de la santé et de la sécurité du travail: 1-36.

56. MacDonald, C., M. MacDonald, and W. Norman, Charitable conflicts of interest. Journal of Business Ethics, 2002. 39(1-2):67-74.

57. Davis, M. and A. Stark, Conflict of Interest in the Professions. NY: Oxford University Press, 2001.

58. Institute Of Medicine, Conflict of Interest in Medical Research Education and Practice. 2009.

59. Bernat, J.L., Disclosure of conflicts of interest: is transparency clear enough? Neurology Today, 2009. 9(5):4,7.

60. Rodwin, M., Conflicts in managed care. New England Journal of Medicine, 1995. 332:604-7.

61. Azer, L., Tolérance zéro en matière d'inconduite sexuelle chez les professionnels de la santé: utopie ou réalité?, Mémoire, Faculté de droit. 2013, Université de Sherbrooke. 
62. Mitchell, J.M., J.D. Reschovsky, and E.A. Reicherter, Use of physical therapy following total knee replacement surgery: implications of orthopedic surgeons' ownership of physical therapy services. Health Services Research, 2016. 51(15):1838-1857.

63. Grant, R.W., Strings Attached: Untangling the Ethics of Incentives. 2011: Princeton University Press. 200.

64. Lo, B., Commentary: Conflict of interest policies: An opportunity for the medical profession to take the lead. Academic Medicine, 2010. 85(1):9-11.

65. Ordre professionnel de la physiothérapie du Québec (OPPQ), Politiques d'amélioration continue de la compétence (PACC), (Période de référence 2010-2013), Editor. 2010, Document adopté au Conseil d'administration du 19 mars 2010 (Mise à jour le 28 février 2013): Montréal.

66. Moore, D.A. and G. Loewenstein, Self-interest, automaticity, and the psychology of conflict of interest. Social Justice Research, 17(2):189-202.

67. College of physiotherapists of Ontario, Ethics E-Learning Module. 2014.

68. Bion, J., Financial and intellectual conflicts of interest: Confusion and clarity. Current Opinion in Critical Care, 2009. 15(6):583-590.

69. Steinbrook, R., Controlling conflict of interest - proposals from the Institute of Medicine. New England Journal of Medicine, 2009. 360(21):2160-2163.

70. Martin, M.W., Explaining wrongdoing in professions. Journal of Social Philosophy, 1999. 30(2):236-250.

71. Papadakis, M.A., et al., Disciplinary action by medical boards and prior behavior in medical school. New England Journal of Medicine, 2005. 353(25):2673-2682.

72. Papadakis, M.A., et al., Unprofessional behavior in medical school is associated with subsequent disciplinary action by a state medical board. Academic Medicine, 2004. 79(3):244249.

73. Papadakis, M.A., et al., Performance during internal medicine residency training and subsequent disciplinary action by state licensing boards. Annals of Internal Medicine, 2008. 148(11):869-876.

74. Laliberté, M. and J.-O. Dyer, L'utilisation du TENS en physiothérapie : la situation particulière des soins palliatifs oncologiques. BioéthiqueOnline, 2012. 1(20).

75. McKneally, M.F., et al., Bioethics for clinicians: resource allocation. Canadian Medical Association Journal, 1997. 157(2):163-167.

76. Hass, J.F., Admission to rehabilitation centers: selection of patients. Archives of Physical Medicine Rehabilitation, 1988. 69(5):329-33.

77. Nunes, R., Evidence-based medicine: a new tool for resource allocation? Medicine, Health Care and Philosophy, 2003. 6(3):297-301.

78. Gibson, J.L., D.K. Martin, and P.A. Singer, Setting priorities in health care organization: criteria, processes, and parameters of success. BMC Heath Services Research, 2004. 4(25).

79. Gibson, J.L., D.K. Martin, and P.A. Singer, Evidence, economics and ethics: resource allocation in health services organizations. Healthcare Quarterly, 2005. 8(2):50-9.

80. Raymond, M.H., et al., Who's next? Referral prioritisation criteria for home care occupational therapy. International Journal of Therapy and Rehabilitation, 2013. 20(12):580-586.

81. Pearsall, M.J. and A.P.J. Ellis, Thick as thieves: the effects of ethical orientation and psychological safety on unethical team behavior. Journal of Applied Psychology, 2010. 96(2):401-11.

82. Kish-Gephart, J.J., D.A. Harrison, and L.K. Trevino, Bad apples, bad cases, and bad barrels: meta-analytic evidence about sources of unethical decisions at work. Journal of Applied Psychology, 2010. 95(1): 1-31.

83. Laliberté, M. and A. Hudon, Do conflicts of interest create a new professional norm? Physical therapists and workers's compensation. The American Journal of Bioethics, 2013. 13(10):2628. 